\title{
Monitoramento remoto para o enfrentamento da obesidade por profissionais de educação física: uma revisão integrativa
}

Remote monitoring to address obesity by physical education professionals: an integrative review

Monitoreo remoto para abordar la obesidad por profesionales de la educación física: una revisión integrativa

Heloá Costa Borim Christinelli ORCID: https://orcid.org/0000-0003-0772-4194

Universidade Estadual de Maringá, Brasil E-mail: heloa.borim@hotmail.com

Wesley Gabriel da Silva Alexandrino ORCID: https://orcid.org/0000-0001-9573-918X Universidade Estadual do Paraná, Brasil E-mail: wesgabriell@outlook.com

Greice Westphal

ORCID: https://orcid.org/0000-0001-9107-0108 Universidade Estadual de Maringá, Brasil E-mail: greicewes@gmail.com Raquel Tomiazzi Utrila

ORCID: https://orcid.org/0000-0001-9454-2713

Universidade Estadual de Maringá, Brasil E-mail: raquelutrila@gmail.com

Maria Luiza Costa Borim

ORCID: https://orcid.org/0000-0002-9523-4218

Universidade Estadual de Maringá, Brasil E-mail: luborim10@hotmail.com

Suelen Cristina Zandonadi Bernal ORCID: https://orcid.org/0000-0002-1327-9261

Universidade Estadual de Maringá, Brasil E-mail: suelenbernal_85@hotamil.com

Igor Alisson Spagnol Pereira ORCID: https://orcid.org/0000-0001-7340-3909

Universidade Estadual de Maringá, Brasil E-mail: igorspagnol2@hotmail.com

Fernando Malentaqui Martins

ORCID: https://orcid.org/0000-0003-1623-2183

Universidade Estadual de Maringá, Brasil E-mail: nandoesporte1@gmail.com

Mario Moreira Castilho

ORCID: https://orcid.org/0000-0002-4855-8236

Universidade Estadual de Maringá, Brasil

E-mail: mmcastilho_1905@hotmail.com

Geison Schmidt Soares

ORCID: https://orcid.org/0000-0001-5150-6931 Universidade Estadual de Maringá, Brasil E-mail: geisonssoares@gmail.com

Michele Fernandes do Amaral

ORCID: https://orcid.org/0000-0002-4812-9773

Universidade Estadual de Maringá, Brasil

E-mail: micheleamaralpsico@gmail.com

Maria Antonia Ramos Costa

ORCID: https://orcid.org/0000-0001-6906-5396

Universidade Estadual do Paraná, Brasil

E-mail: maria.costa@unespar.edu.br

Nelson Nardo Junior

ORCID: https://orcid.org/0000-0002-6862-7868

Universidade Estadual de Maringá, Brasil

E-mail: nnjunior@uem.br 
Carlos Alexandre Molena Fernandes

ORCID: https://orcid.org/0000-0002-4019-8379 Universidade Estadual de Maringá, Brasil E-mail: carlosmolena126@gmail.com

\begin{abstract}
Resumo
Introdução: O mundo encontra-se em situação de pandemia por COVID-19, uma doença infecciosa que afeta seres humanos causada pelo coronavírus, denominado SARS-CoV-2. Considerando o aumento da obesidade durante a pandemia na população brasileira, é necessário ampliar a capacidade de assistência do educador físico, buscando novas estratégias de promoção da saúde por meio de orientações para mudança nos hábitos de vida. Objetivo: Identificar quais tipos de monitoramento remoto são utilizados para a realização de exercício físico remoto em obesos. Método: Trata-se de uma revisão integrativa da literatura realizada durante o mês de junho de 2020 na base de dados National Library of Medicine (PubMed), utilizando os seguintes descritores: "Obesidade", "Tecnologia Biomédica", "Aplicativos móveis", "Promoção da saúde", "Prevenção de doenças", "Telemonitoramento", "Exercício físico". Os critérios de inclusão definidos para a seleção dos artigos foram: artigos originais, revisões de literatura (sistemática, integrativa ou narrativa) e relatos de experiência, publicados entre abril de 2014 a abril de 2020, nos idiomas inglês e português, disponíveis na íntegra e que apresentassem a descrição das etapas/métodos utilizados para a intervenção com o exercício físico remoto por educadores físicos. Os critérios de exclusão foram: duplicidade dos artigos, editoriais, anais de congresso, estudo de caso e artigos de reflexão; intervenção realizada por outro profissional. Resultados: Os tipos de monitoramento remoto mais utilizados por educadores físicos no tratamento da obesidade foram: aplicativos para smartphone, mensagens de texto, e-mails, cartas (enviadas por correio) e site. Conclusão: Intervenções remotas mostram-se eficazes para perda de peso no curto prazo. Além da perda de peso o monitoramento remoto é favorável para o aumento da frequência de realização de atividade física e outras mudanças comportamentais em obesos.
\end{abstract}

Palavras-chave: Obesidade; Tecnologia biomédica; Aplicativos móveis; Promoção da saúde; Prevenção de doenças; Telemonitoramento; Exercício físico.

\begin{abstract}
Introduction: The world is in a pandemic situation due to COVID-19, an infectious disease that affects humans caused by the coronavirus, called SARS-CoV-2. Considering the increase in obesity during the pandemic in the Brazilian population, it is necessary to expand the capacity to assistance from the physical educator, seeking new health promotion strategies through guidelines for changing lifestyle habits. Objective: To identify which types of remote monitoring are used to perform remote physical exercise in obese individuals. Method: This is an integrative literature review carried out during the month of June 2020 in the National Library of Medicine database (PubMed), using the following descriptors: "Obesity", "Biomedical Technology", "Mobile applications", "Health promotion", "Disease prevention", "Telemonitoring", "Physical exercise". The inclusion criteria defined for the selection of articles were: original articles, literature reviews (systematic, integrative or narrative) and experience reports, published between April 2014 and April 2020, in English and Portuguese, available in full and that presented the description of the steps / methods used for the intervention with remote physical exercise by physical educators. The exclusion criteria were: duplicity of articles, editorials, conference proceedings, case study and reflection articles; intervention by another professional. Results: The types of remote monitoring most used by physical educators in the treatment of obesity were: smartphone apps, text messages, e-mails, letters (sent by mail) and website. Conclusion: Remote interventions are effective for weight loss in the short term. In addition to weight loss, remote monitoring is favorable for increasing the frequency of physical activity and other behavioral changes in obese individuals.
\end{abstract}

Keywords: Obesity; Biomedical technology; Mobile apps; Health promotion; Prevention of diseases; Telemonitoring; Physical exercise.

\title{
Resumen
}

Introducción: El mundo se encuentra en una situación de pandemia por COVID-19, una enfermedad infecciosa que afecta a los humanos causada por el coronavirus, llamado SARS-CoV-2. Considerando el aumento de la obesidad durante la pandemia en la población brasileña, es necesario ampliar la capacidad asistencial del educador físico, buscando nuevas estrategias de promoción de la salud a través de pautas para el cambio de hábitos de vida. Objetivo: Identificar qué tipos de monitorización remota se utilizan para realizar ejercicio físico a distancia en personas obesas. Método: Se trata de una revisión integradora de la literatura realizada durante el mes de junio de 2020 en la base de datos de la Biblioteca Nacional de Medicina (PubMed), utilizando los siguientes descriptores: "Obesidad", "Tecnología Biomédica", "Aplicaciones móviles", "Promoción de la salud", "Prevención de enfermedades", "Telemonitorización", "Ejercicio físico". Los criterios de inclusión definidos para la selección de artículos fueron: artículos originales, revisiones de la literatura (sistemática, integradora o narrativa) e informes de experiencia, publicados entre abril de 2014 y abril de 2020, en inglés y portugués, disponibles en su totalidad y que presentó la descripción de los pasos / métodos utilizados para la intervención con ejercicio físico a distancia por parte de los educadores físicos. Los criterios de exclusión fueron: duplicidad de artículos, editoriales, actas de congresos, estudios 
de caso y artículos de reflexión; intervención de otro profesional. Resultados: Los tipos de monitoreo remoto más utilizados por los educadores físicos en el tratamiento de la obesidad fueron: aplicaciones para teléfonos inteligentes, mensajes de texto, correos electrónicos, cartas (enviadas por correo) y sitio web. Conclusión: Las intervenciones a distancia son efectivas para la pérdida de peso a corto plazo. Además de la pérdida de peso, la monitorización remota es favorable para aumentar la frecuencia de la actividad física y otros cambios de comportamiento en las personas obesas.

Palabras clave: Obesidad; Tecnología biomédica; Aplicaciones móviles; Promoción de la salud; Prevención de enfermedades; Telemonitorización; Ejercicio físico.

\section{Introdução}

O mundo encontra-se em situação de pandemia por COVID-19, uma doença infecciosa que afeta seres humanos causada pelo coronavírus, denominado SARS-CoV-2, descoberto recentemente. Este novo vírus e doença era desconhecido antes do início do surto em Wuhan na China, em dezembro de 2019 (WHO, 2020).

Dentre os fatores de risco para desenvolvimento da forma grave da doença e óbito, a obesidade tem merecido papel de destaque. Estudo realizado em 181 países com 4.670.832 casos de COVID-19 e 311.384 mortes, observou que a obesidade é o principal fator de risco modificável associado à infecção por SARS-CoV-2 (Burden et al., 2020).

Além disso, tem sido verificado em pessoas com excesso de peso que o risco de complicações graves pela COVID-19 é maior quando comparado com sujeitos eutróficos (Finer et al., 2020), isto por que as consequências fisiológicas da obesidade como, por exemplo, a inflamação, contribuem para o desenvolvimento da doença e a gravidade dos casos nesta população (Caci et al., 2020). Pesquisa realizada com 4.103 pacientes com doença de COVID - 19 na cidade de Nova York, identificou que os aspectos clínicos mais importantes que levaram à internação foram idade superior a 65 anos e a obesidade, mais do que hipertensão, diabetes ou doença cardiovascular (Petrilli et al., 2020).

Vale ressaltar que no Brasil a prevalência da obesidade em adultos passou de 11,8\% em 2016, para 20,3\% em 2019, isso significa que dois em cada dez brasileiros estão obesos (Brasil, 2019), dado importante se observarmos que 20,3\% da população brasileira tem maior risco de agravamento do quadro de COVID-19, apenas por serem obesas.

O aumento da prevalência da obesidade está relacionado aos efeitos negativos da urbanização rápida e da globalização, que induzem a maioria das pessoas ao estilo de vida sedentário, além de promover mudanças no padrão alimentar da população, ou seja, o aumento no consumo de produtos ultra processados, com altos níveis de sódio, açúcar e gorduras saturadas (Monteiro, 2018; WHO 2015).

Além destas mudanças já ocorridas nos hábitos e estilos de vida, com a pandemia do novo coronavírus, a população foi aconselhada a manter o distanciamento social como meio básico de limitar a exposição da população ao vírus, como consequência do isolamento social, as restrições impostas na população inevitavelmente interromperão as atividades diárias de milhões de pessoas, como por exemplo, atividade física regular e exercícios (Mera et al., 2020).

$\mathrm{O}$ isolamento social embora seja uma medida de segurança, pode ter consequências negativas não intencionais, uma vez que tais esforços para evitar a transmissão do vírus entre humanos podem aumentar os comportamentos que levam à inatividade física e redução de alimentos saudáveis por alimentos ultra processados, em decorrência das restrições sociais impostas (Chen et al., 2020; Malta et al., 2020).

Ou seja, é importante incorporar estratégias relacionadas a atividades física, bem como recomendações nutricionais e repouso adequado, a aplicação dessas estratégias podem trazer benefícios imediatos na melhoria da qualidade de vida e redução dos riscos de infecções (Castilho, 2021; Christinelli et al., 2021; Mera et al., 2020; Westphal et al., 2021).

Neste cenário, o profissional de educação física tem um papel relevante visto que suas ações permitem a realização de práticas assistenciais que promovam a prevenção e o tratamento da obesidade (Castilho et al., 2021; 
Christinelli et al., 2020; Pereira et al., 2021; Westphal et al., 2020). Recomenda-se que indivíduos de 18 a 64 anos realizem pelo menos 150 minutos de atividade física por semana, tendo em vista benefícios significativos para a saúde e a prevenção de Doenças Crônicas Não Transmissíveis (DCNTs) (WHO, 2018).

Considerando o aumento progressivo da frequência da obesidade na população brasileira, é necessário ampliar a capacidade de assistência do educador físico, sendo assim, faz-se necessário buscar novas estratégias de promoção da saúde por meio de orientações para mudança nos hábitos de vida da população (Castilho et al., 2021; De Alves et al., 2016; Westphal et al., 2020).

Visando a ampliação da assistência do profissional de educação física, nos últimos anos estão sendo utilizados programas para o monitoramento remoto da saúde para assistência da população, alguns desses programas de intervenção de saúde para adultos foram conduzidos para perder peso corporal, promover a saúde e melhorar os hábitos alimentares (Kim \& Seo, 2020; Pereira et al., 2021).

O monitoramento remoto é um meio promissor para promover mudanças no comportamento entre pessoas com doenças crônicas, fornecendo-lhes informações de saúde e sugestões oportunas para melhorar os comportamentos de saúde, feedbacks e apoio social (Wang et al., 2017). Sendo que durante a pandemia o monitoramento remoto tornou-se um componente crítico para aumentar a capacidade de combater o coronavírus, e ao mesmo tempo, manter os serviços de saúde funcionastes e mais seguros (Mera et al., 2020).

Nesse sentido, questiona-se "Como tem sido realizado o monitoramento de exercício físico remoto em indivíduos obesos?" Este estudo teve como objetivo identificar quais tipos de monitoramento remoto são utilizados para realização de exercício físico remoto em pessoas com obesidade.

\section{Metodologia}

Trata-se de um estudo com coleta de dados realizada a partir de fontes secundárias, por meio de uma revisão integrativa. A pesquisa bibliográfica é uma das melhores formas de iniciar um estudo, buscando-se semelhanças e diferenças entre os artigos levantados nos documentos de referência (Soares et al., 2010).

A revisão da literatura foi realizada no mês de Junho de 2020, na base de dados National Library of Medicine (PubMed), utilizando para busca de artigos na língua portuguesa os seguintes descritores, extraídos dos Descritores em Ciências da Saúde (DeCs): "Obesidade", "Tecnologia Biomédica", "Aplicativos móveis", "Promoção da saúde", "Prevenção de doenças", "Telemonitoramento", "Exercício físico"; enquanto que para a busca dos artigos da língua inglesa, foram utilizados os descritores equivalentes extraídos do Medical Subject Headings (MeSH): "Obesity", "Biomedical technology", "Mobile Applications" "Health promotion", "Disease prevention", Telemonitoring", e "Exercise".

Os critérios de inclusão definidos para a seleção dos artigos foram: artigos originais, revisões de literatura (sistemática, integrativa ou narrativa) e relatos de experiência, publicados entre abril de 2014 a abril de 2020, nos idiomas inglês e português, disponíveis na íntegra e que apresentassem a descrição das etapas/métodos utilizados para a intervenção com o exercício físico remoto realizados por educadores físicos. Os critérios de exclusão foram: Duplicidade dos artigos, editoriais, anais de congresso, estudo de caso e artigos de reflexão; intervenção realizada por outro profissional. A análise dos artigos se baseou na seguinte questão de pesquisa: "Como é realizado o monitoramento de exercício físico remoto em indivíduos obesos?".

Foram coletadas as seguintes informações para análise: título do artigo, autores, base de dados, ano de publicação, país do estudo, Qualis Capes, tipo de pesquisa, característica da amostra, tamanho da amostra, analise estatística, nível de significância, intervenção realizada, presença de grupo controle, duração da intervenção, 
responsáveis pela intervenção, como foi realizada a avaliação da intervenção, resultados, conclusão, recomendações dos autores, clareza na identificação da trajetória metodológica no texto e identificação de limitações de viés. Estas informações foram organizadas em uma tabela do software Excel® e analisadas de forma descritiva.

A busca na base de dados retornou um total de 94 artigos, desses artigos 63 foram selecionados para a leitura dos títulos e resumo, após a leitura 57 artigos foram considerados inelegíveis e 6 artigos foram selecionados para a análise.

O resultado das análises foi realizado de forma descritiva, com análise crítica das informações encontradas.

\section{Resultados}

A amostra final dessa revisão integrativa resultou em seis artigos científicos. A origem dos artigos e a classificação das publicações são apresentados no Quadro 1.

Quadro 1. Identificação dos estudos analisados na revisão integrativa da literatura, disponíveis em Junho de 2020.

\begin{tabular}{|l|c|c|c|}
\hline \multicolumn{1}{|c|}{ Título } & País & Idioma & Qualis Capes \\
\hline $\begin{array}{l}\text { Telemonitoring-supported exercise training, metabolic } \\
\text { syndrome severity, and work ability in company employees: a } \\
\text { randomised controlled trial. }\end{array}$ & Alemanha & Inglês & A1 \\
\hline $\begin{array}{l}\text { Using Social and Mobile Tools for Weight Loss in } \\
\text { Overweight and Obese Young Adults (Project SMART): A 2- } \\
\text { Year Parallel Group Randomized Controlled Trial. }\end{array}$ & EUA & Inglês & A1 \\
\hline $\begin{array}{l}\text { Smartphone-Based Health Program for Improving Physical } \\
\text { Activity and Tackling Obesity for Young Adults: A } \\
\text { Systematic Review and Meta-Analysis }\end{array}$ & EUA & Inglês & B1 \\
\hline $\begin{array}{l}\text { Long-Term Follow-Up of the Telemonitoring Weight- Reduction } \\
\text { Program (Active Body Control). }\end{array}$ & Alemanha & Inglês & A2 \\
\hline $\begin{array}{l}\text { Efficacy of a Mobile Social Networking Intervention in } \\
\text { Promoting Physical Activity: Quasi-Experimental Study. }\end{array}$ & Austrália & Inglês & A3 \\
\hline $\begin{array}{l}\text { Successful weight reduction and maintenance by using a } \\
\text { smartphone application in those with overweight and obesity. }\end{array}$ & EUA & Inglês & A1 \\
\hline
\end{tabular}

Fonte: Autores.

O Quadro 2 apresenta as características dos estudos selecionados. 
Quadro 2. Métodos e intervenções utilizados pelos estudos selecionados na revisão integrativa da literatura, disponíveis em Junho de 2020.

\begin{tabular}{|c|c|c|c|c|}
\hline Autores & Título & Tipo de estudo & Amostra & Intervenção realizada \\
\hline $\begin{array}{l}\text { HAUFE, S. } \\
\text { et al. }\end{array}$ & $\begin{array}{l}\text { Telemonitoring- } \\
\text { supported } \\
\text { exercise } \\
\text { training, } \\
\text { metabolic } \\
\text { syndrome } \\
\text { severity, and } \\
\text { work ability in } \\
\text { company } \\
\text { employees: a } \\
\text { randomised } \\
\text { controlled trial }\end{array}$ & $\begin{array}{l}\text { Estudo } \\
\text { prospectivo }\end{array}$ & 264 & $\begin{array}{l}\text { Uma intervenção no estilo de vida de } 6 \text { meses } \\
\text { com foco em exercícios regulares (grupo de } \\
\text { exercícios) ou para uma lista de espera grupo de } \\
\text { controle, usando uma lista de atribuição baseada } \\
\text { em computador com comprimento de bloco } \\
\text { variável. Os participantes do grupo de exercícios } \\
\text { receberam recomendações individuais de } \\
\text { exercícios em encontros presenciais e por meio } \\
\text { de um aplicativo de smartphone, com o objetivo } \\
\text { de realizar } 150 \text { min de atividade física por } \\
\text { semana. As atividades foram supervisionadas e } \\
\text { adaptadas usando dados do monitor de } \\
\text { atividades, que foram transferidos para um banco } \\
\text { de dados central. Os participantes do grupo de } \\
\text { controle continuaram com seu estilo de vida atual } \\
\text { e foram informados sobre a possibilidade de } \\
\text { receber a intervenção supervisionada após a } \\
\text { conclusão do estudo }\end{array}$ \\
\hline $\begin{array}{l}\text { GODINO, } \\
\text { J.G. et al. }\end{array}$ & $\begin{array}{l}\text { Using Social } \\
\text { and Mobile } \\
\text { Tools for Weight } \\
\text { Loss in } \\
\text { Overweight and } \\
\text { Obese Young } \\
\text { Adults (Project } \\
\text { SMART): A 2- } \\
\text { Year Parallel } \\
\text { Group } \\
\text { Randomized } \\
\text { Controlled Trial. }\end{array}$ & $\begin{array}{l}\text { Estudo } \\
\text { controlado } \\
\text { randomizado }\end{array}$ & 404 & $\begin{array}{l}\text { A intervenção SMART foi teoricamente } \\
\text { informada pela taxonomia de Abraham e Michie } \\
\text { de mudança de comportamento. A intervenção } \\
\text { SMART foi entregue remotamente por meio de } \\
\text { seis modalidades: Facebook, três aplicativos } \\
\text { moveis planejados para o estudo, mensagens de } \\
\text { texto, e-mails, um site e comunicação mediana } \\
\text { por tecnologia com um profissional de saúde } \\
\text { coach. Os participantes foram instruídos a usar } \\
\text { pelo menos uma ou mais modalidades no mínimo } \\
\text { cinco vezes por semana durante os } 24 \text { meses. Os } \\
\text { participantes da intervenção puderam definir de } \\
\text { forma privada ou publica, as atividades e metas } \\
\text { de dietas individualizadas e em seguida, escolher } \\
\text { sua modalidade preferida e monitorar esses } \\
\text { comportamentos, receber feedback e participar } \\
\text { da revisão de metas. }\end{array}$ \\
\hline $\begin{array}{l}\text { KIM, H. N. } \\
\text { et al. }\end{array}$ & $\begin{array}{l}\text { Smartphone- } \\
\text { Based Health } \\
\text { Program for } \\
\text { Improving } \\
\text { Physical Activity } \\
\quad \text { and } \\
\text { Tackling Obesity } \\
\quad \text { for } \\
\text { Young Adults: A } \\
\text { Systematic and } \\
\text { Review Meta-Analysis }\end{array}$ & $\begin{array}{l}\text { Revisão } \\
\text { sistemática da } \\
\text { literatura } \\
\text { metanálise, }\end{array}$ & 1830 & $\begin{array}{l}\text { Os programas de intervenção consistiram em } \\
\text { intervenção no estilo de vida, educação e } \\
\text { treinamento para promover atividade } \\
\text { física, aconselhamento dietético, educação } \\
\text { relacionada ao comportamento em saúde e } \\
\text { feedback, que foram fornecidos de } 5 \text { a } 24 \text { vezes } \\
\text { ao longo de dois a seis meses. Ao usar } \\
\text { aplicativos para smartphone como estratégia de } \\
\text { intervenção, os sujeitos podiam registrar } \\
\text { diretamente seu estado de saúde e nutrição e } \\
\text { receber feedback. Foram enviadas mensagens de } \\
\text { texto ou e-mails personalizados pelas } \\
\text { informações fornecidas por um sujeito ou } \\
\text { chamadas de educação e treinamento } \\
\text { personalizadas, dependendo do seu estado de } \\
\text { saúde e nutrição. Em alguns casos, foram } \\
\text { fornecidos aplicativos para smartphones e } \\
\text { educação adicional baseada na Web, além de } \\
\text { dispositivos para verificar a educação em estilo } \\
\text { de palestra e os passos dados. No estudo que } \\
\text { forneceu um dispositivo de contagem de etapas. }\end{array}$ \\
\hline
\end{tabular}




\begin{tabular}{|c|c|c|c|c|}
\hline $\begin{array}{l}\text { STUMM, G. } \\
\text { et al. }\end{array}$ & $\begin{array}{l}\text { Long-Term } \\
\text { Follow-Up of } \\
\text { the } \\
\text { Telemonitoring } \\
\text { Weight- } \\
\text { Reduction } \\
\text { Program (Active } \\
\text { Body } \\
\text { Control). }\end{array}$ & $\begin{array}{l}\text { Estudo } \\
\text { controlado } \\
\text { randomizado }\end{array}$ & 49 & $\begin{array}{l}\text { O programa de redução de peso Active Body } \\
\text { Control (ABC) é baseado no telemonitoramento } \\
\text { da atividade física e nutrição em conjunto com a } \\
\text { tele- abordagem por meio de cartas de } \\
\text { aconselhamento semanais enviadas por correio } \\
\text { ou por e-mail. Os participantes que completaram } \\
\text { os programas por um ano foram randomizados } \\
\text { em dois grupos, O grupo "ABC continuado" } \\
\text { manteve o telemonitoramento e recebeu } \\
\text { teleconferência pelo segundo ano e foi } \\
\text { aconselhado por cartas mensais, O grupo } \\
\text { "descontinuado ABC" não foi } \\
\text { contatado por mais } 12 \text { meses até o exame médico } \\
\text { após } 24 \text { meses. }\end{array}$ \\
\hline $\begin{array}{l}\text { TONG, H.L. } \\
\text { et al. }\end{array}$ & $\begin{array}{l}\text { Efficacy of a } \\
\text { Mobile Social } \\
\text { Networking } \\
\text { Intervention in } \\
\text { Promoting } \\
\text { Physical } \\
\text { Activity: Quasi- } \\
\text { Experimental } \\
\text { Study. }\end{array}$ & $\begin{array}{l}\text { Quase } \\
\text { experimental }\end{array}$ & 55 & $\begin{array}{l}\text { A intervenção envolveu } 3 \text { componentes, } \\
\text { incluindo um aplicativo móvel (denominado } \\
\text { Fit.healthy.me), um rastreador vestível e } \\
\text { mensagens de texto e e-mail de mensagens curtas, } \\
\text { (SMS). O aplicativo Fit.healthy.me foi } \\
\text { desenvolvido com base em várias técnicas de } \\
\text { comportamento, comportamento, como o } \\
\text { automonitoramento da atividade física, suporte } \\
\text { social e comparação social. Para permitir a } \\
\text { automação do automonitoramento, o aplicativo } \\
\text { foi integrado ao rastreador vestível Fitbit Flex } 2, \\
\text { esse rastreador usa tecnologia de acelerômetro } \\
\text { para medir aceleração, que são convertidas e, } \\
\text { contagem de passos - um indicador comum de } \\
\text { atividade física. Além disso, avisos e dicas eram } \\
\text { enviados a cada } 2 \text { semanas para lembrar os } \\
\text { usuários de usar o rastreador fitness durante as } \\
\text { horas de vigília. }\end{array}$ \\
\hline $\begin{array}{l}\text { CHIN, S. O. } \\
\text { et al. }\end{array}$ & $\begin{array}{l}\text { Successful } \\
\text { weight reduction } \\
\text { and maintenance } \\
\text { by using a } \\
\text { smartphone } \\
\text { application in } \\
\text { those with } \\
\text { overweight and } \\
\text { obesity. }\end{array}$ & Retrospectivo & 3500 & $\begin{array}{l}\text { Noom Coach é um dos aplicativos mais } \\
\text { populares disponíveis para a perda de peso. No } \\
\text { primeiro login, os usuários são solicitados a } \\
\text { definir seu peso corporal alvo e registrar seu peso } \\
\text { corporal atual. Ao usar o aplicativo, os usuários } \\
\text { registram sua ingestão diária de alimentos e um } \\
\text { monitor de atividade embutido no aplicativo } \\
\text { salva diariamente o número de passos dados por } \\
\text { dia. Com base nesses dados, o aplicativo gera } \\
\text { relatórios que demostram as tendências de peso } \\
\text { do usuário, bem como resumos calóricos e } \\
\text { nutricionais de sua dieta e fornece feedback, } \\
\text { incluindo os tipos de exercícios que ajudam a } \\
\text { atingir o peso corporal desejado do usuário. }\end{array}$ \\
\hline
\end{tabular}

Fonte: Autores.

O Quadro 3 apresenta os principais resultados das intervenções de monitoramento remoto. 
Quadro 3 - Resultados das intervenções realizadas pelos estudos selecionados na revisão integrativa da literatura, disponíveis em junho de 2020.

\begin{tabular}{|c|c|}
\hline Título & Resultados das intervenções \\
\hline $\begin{array}{l}\text { Telemonitoring- supported } \\
\text { exercise training, metabolic } \\
\text { syndrome severity, and } \\
\text { work ability in company } \\
\text { employees: a randomised } \\
\text { controlled trial. }\end{array}$ & 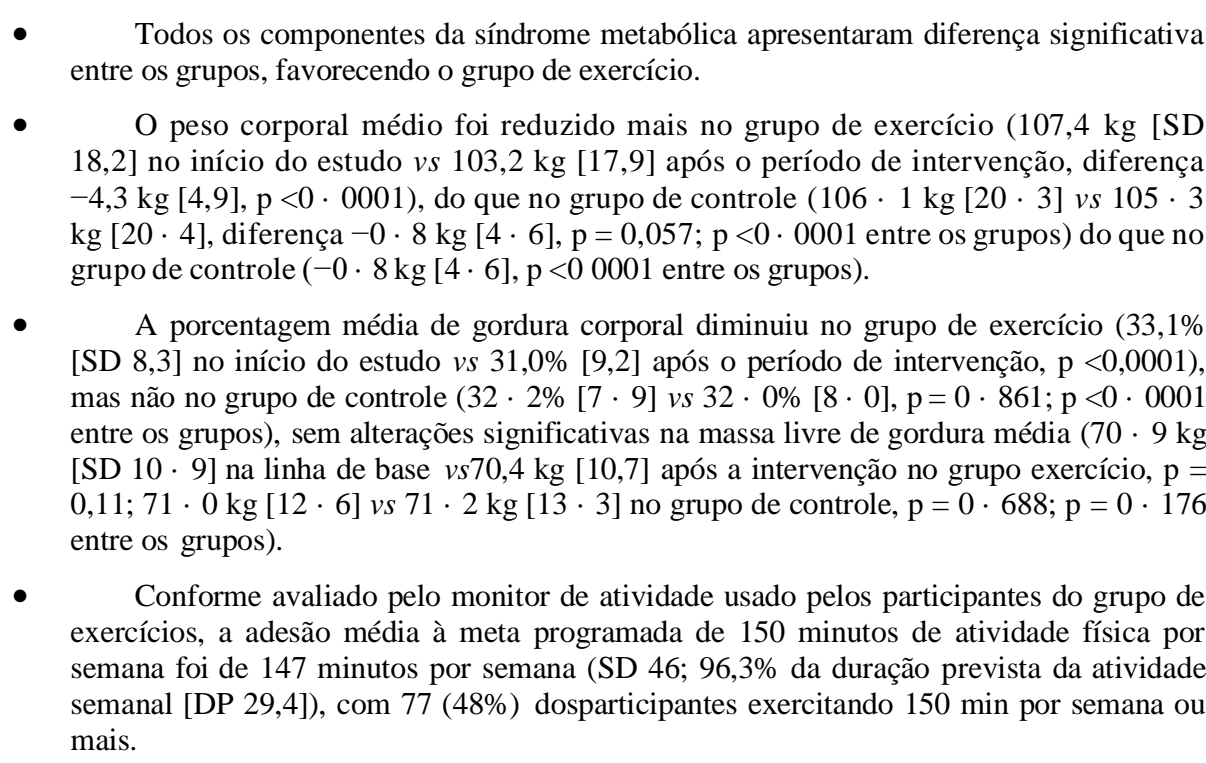 \\
\hline $\begin{array}{l}\text { Using Social and Mobile } \\
\text { Tools for Weight Loss in } \\
\text { Overweight and Obese } \\
\text { Young Adults (Project } \\
\text { SMART): A2-Year Parallel } \\
\text { Group Randomized } \\
\text { Controlled Trial. }\end{array}$ & $\begin{array}{l}\text { - O peso ajustado por sexo, etnia e faculdade foi significativamente menor no grupo de } \\
\text { intervenção SMART em comparação com o grupo controle em } 6 \text { meses }(-1,33 \mathrm{~kg}, \mathrm{p}= \\
0,011) \text { e } 12 \text { meses }(-1.33 \mathrm{~kg}, \mathrm{p}=0,008) \text {. } \\
\text { - As diferenças entre os grupos aos } 18 \text { meses }(-0,67 \mathrm{~kg}, \mathrm{p}=0,200) \text { e } 24 \text { meses }(-0,79 \mathrm{~kg} \text {, } \\
\mathrm{p}=0,204) \text { não foram estatisticamente significativas. } \\
\text { As diferenças no IMC entre grupo de intervenção SMART e o grupo de controle } \\
\text { - paralelamente ao peso em que as diferenças foram significativas apenas em } 6 \text { a } 12 \text { meses, } \\
\text { diferenças pequenas mas estatisticamente significantes entre os grupos na proporção de } \\
\text { participantes que perderam } 5 \% \text { do peso corporal e circunferência da cintura em } 6 \text { meses. }\end{array}$ \\
\hline $\begin{array}{l}\text { Smartphone-Based Health } \\
\text { Program for Improving } \\
\text { Physical Activity and } \\
\text { Tackling Obesity for Young } \\
\text { Adults: A Systematic } \\
\text { Reviewand Meta- } \\
\text { Analysis }\end{array}$ & $\begin{array}{l}\text { Diferenças significativas nos resultados entre os grupos de intervenção e controle } \\
\text { - foram encontradas para atividade física (um estudo), e peso relatado (um estudo). } \\
\text { A diferença média padrão (SMD) dos cinco artigos }(\mathrm{n}=1830) \text { sobre a mudança na } \\
\text { atividade física foi de } 2,59\left(\mathrm{I}^{2}=99 \%, p=0,001 \text {, intervalo de confiança: } 1,00,4,18\right) \\
\text { indicando um aumentosignificativo na atividade física nos grupos de intervenção. } \\
\text { - A diferença média combinada dos três artigos sobre mudança de peso foi }-2,80\left(\mathrm{I}^{2}=\right. \\
0 \%, p=0,002, \mathrm{IC}:-4,54,-1,06), \text { o que sugeriu perda de peso significativa nos grupos de } \\
\text { intervenção. }\end{array}$ \\
\hline $\begin{array}{l}\text { Long-Term Follow-Up } \\
\text { of the } \\
\text { Telemonitoring Weight- } \\
\text { Reduction Program } \\
\text { (Active } \\
\text { Body Control). }\end{array}$ & $\begin{array}{l}\text { - As mudanças de peso relativo após o primeiro ano foram, respectivamente, }-13,4 \% \text { e } \\
-11,4 \% \text { nos grupos "ABC descontinuado" e "ABC continuado", e após o segundo ano } \\
\text { diminuíram, respectivamente, } 4,4 \text { e } 2,8 \% \text {. No entanto, essa diferença no ganho de peso } \\
\text { entre os dois grupos não foi estatisticamente significativa. }\end{array}$ \\
\hline $\begin{array}{l}\text { Efficacy of a Mobile Social } \\
\text { Networking Intervention in } \\
\text { Promoting Physical } \\
\text { Activity: } \\
\text { Experimental Study }\end{array}$ & $\begin{array}{l}\text { Um total de } 55 \text { participantes foram incluídos no estudo; a média de idade foi de } 23,6 \\
\text { anos e } 28(51 \%) \text { eram do sexo feminino. Houve um aumento não estatisticamente } \\
\text { significativo na contagem média de passos diários entre a linha de base e } 6 \text { meses } \\
\text { (mudança média }=14,5 \text { passos / dia, } P=0,98 \text {, IC } 95 \%-1136,5 \text { a } 1107,5 \text { ). A } \\
\text { análise de subgrupo comparando os grupos de atividade física superior e inferior } \\
\text { no início do estudo mostrou que o último teve um aumento estatisticamente } \\
\text { significativamente maior em sua contagem de passos diários (diferença do grupo } \\
\text { na mudança média desde o início até } 6 \text { meses = } 3025 \text { passos por dia, } P=0,008 \text {, } \\
95 \% \text { CI 837,9-5211,8). Aos } 6 \text { meses, a taxa de retenção foi de } 82 \%(45 / 55) \text {; o } \\
\text { uso do aplicativo diminuiu com o tempo. }\end{array}$ \\
\hline
\end{tabular}




\begin{tabular}{|l|l|l|}
\hline $\begin{array}{l}\text { Successful weight } \\
\text { reduction and maintenance } \\
\text { by using a smartphone } \\
\text { application in those with } \\
\text { overweight and }\end{array}$ & $\begin{array}{l}\text { Entre os participantes, 77,9\% relataram uma diminuição no peso corporal enquanto } \\
\text { usavam o aplicativo, com 22,7\% experimentando mais de } 10 \% \text { de perda de peso em } \\
\text { comparação com a linha de base, com uma taxa de sucesso de perda de peso mais alta em } \\
\text { homens }(83,9 \mathrm{vs} .76,1 \%) \text {, resultando em IMC final de } 28,1 \pm 0,1 \mathrm{~kg} / \mathrm{m}^{2} \mathrm{para} \text { homens e } \\
\text { obesity. }\end{array}$ \\
$\begin{array}{l}26,5 \pm 0,0 \mathrm{~kg} / \mathrm{m}^{2} \text { para mulheres. } \\
\end{array}$
\end{tabular}

Fonte: Autores.

\section{Discussão}

O desenvolvimento de ações durante a pandemia para a população e comunidade por profissionais da saúde, visam a diminuição drástica da população que seriam encaminhadas, desnecessariamente a atenção primária à saúde, introduzindo o uso de tecnologias de informações e comunicações (TICs )(IBGE, 2018) para o monitoramento da população (Harzheim et al., 2020). O monitoramento remoto é uma ferramenta com objetivo de ampliar o acesso aos cuidados em saúde, tendo se mostrado um recurso eficiente para o atendimento da população, possibilitando o atendimento remoto com a mesma qualidade que o presencial (Dimer et al., 2020).

As principais ferramentas utilizadas para realização do monitoramento remoto pelos educadores físicos foram: aplicativos para smartphone, mensagens de texto, e-mails, cartas (enviadas por correio) e site. Ferramentas que possibilitam o monitoramento remoto de atividade físicas são de suma importância para ampliar o acesso a orientações profissionais na Atenção Primária à saúde, principalmente neste momento de distanciamento social devido à pandemia por COVID-19.

No Brasil 79,1\% da população tem acesso à internet (IBGE, 2018), o que pode gerar bons resultados em intervenções tecnológicas, baseadas em web. Neste sentido, as tecnologias remotas oferecem um novo modo de entrega da atividade física para o usuário, além de ofertar vantagens em termos de uso, alcance e disseminação de recursos, sendo assim essas intervenções tecnológicas podem ser uma forma econômica e viável de atingir a população, de forma mais abrangente, interativa e responsável (Direito et al., 2017).

$\mathrm{O}$ uso de tecnologias tem aumentado à medida que se tornam mais populares e onipresentes, ou seja, as tecnologias estão presentes em todos os lugares do mundo ao mesmo tempo, tornando-se métodos atraentes no domínio da saúde pública, apresentando uma oportunidade de baixo custo pra disseminar informações de saúde de forma viral, possivelmente melhorando o custo-efetividade das intervenções de saúde, podem promover apoio e influencia social, facilitando a mudança de comportamento em saúde (Tong et al., 2019).

Uma revisão sistemática da literatura e meta-análise, mostrou que os programas de saúde para smartphones usado pelos participantes dos artigos analisados apresentam efeitos significativos no aumento da atividade física e na perda de peso (Romeo et al., 2019).

O estudo que obteve melhor resultado foi de Haufe et al (2019) uma intervenção de curto prazo, focada em exercícios e utilizando o monitoramento remoto para a redução da gravidade da síndrome metabólica, a adesão da atividade física dos participantes durante a intervenção foi de 147 minutos por semana, a meta prevista era de 150 minutos por semana e $48 \%$ dos participantes se exercitaram na meta prevista ou mais, e em relação ao peso o resultado foi de uma perda de 4,3 kg comparado com o grupo controle em 6 meses, essa forma de intervenção mostrou um potencial significativo para reduzir o risco de doenças (Haufe et al., 2019).

Comparando com o estudo de Godino et al (2016) uma intervenção com duração de dois anos, o peso perdido nesse período foi de $1,33 \mathrm{~kg}$, sendo assim não teve um resultado significativo na redução de peso, a intervenção obteve resultados não significativos durante esse período, ou seja, intervenções focadas em curto prazo mostram-se mais 
eficazes para a redução de peso através da atividade física. Neste sentido é preciso pensar em soluções para que não haja reganho de peso a longo prazo, de forma que a mudança nos hábitos e estilos de vida seja permanente (Godino et al., 2016).

As intervenções encontradas nesta revisão, estas variaram de 6 meses a 2 anos. Existem estudos que realizaram intervenções remotas com períodos menores (10 semanas) e que já apresentaram mudanças significativas de comportamentos e aumento da atividade física (Flores-Mateo et al., 2015; Schoeppe et al., 2016).

Considerando que é necessária uma mudança efetiva nos hábitos e estilo de vida dos indivíduos para a manutenção dos resultados alcançados nas intervenções, o monitoramento remoto a longo prazo parece ser uma boa alternativa para continuar o estímulo aos hábitos de vida saudáveis. Visto que a falta de estímulos leva o indivíduo a um nível de relaxamento e acomodação, ou seja, é importante que os educadores físicos acompanhem e contribuam no desenvolvimento e manutenção de ações que promovam a adoção de hábitos de vida saudáveis, visto que este profissional tem papel fundamental na promoção da saúde da população (Castilho, 2021; De Alves et al., 2016; Pereira et al., 2021; Westphal et al., 2020).

Neste sentido, o profissional de educação física pode desenvolver atividades na Atenção Primária à Saúde tanto para orientar sobre a importância de hábitos de vida, quanto para promover e estimular a adoção de um estilo de vida ativo, contribuindo para minimizar os riscos de DCNTs (Castilho, 2021; Hartmann \& Lopes, 2020). Para o desenvolvimento destas atividades, o uso de tecnologias móveis podem auxiliar o profissional, uma vez que essas tecnologias são eficientes e apresentam efeito significativo no aumento da atividade física dos indivíduos (Romeo et al., 2019).

\section{Conclusão}

As principais ferramentas utilizadas para realização do monitoramento remoto pelos estudos foram: aplicativos para smartphone, mensagens de texto, e-mails, cartas (enviadas por correio) e site. Ferramentas que possibilitam o monitoramento remoto de atividades físicas são importantes para ampliar o acesso a orientações profissionais na Atenção Primária à Saúde, principalmente neste momento de distanciamento social devido à pandemia por COVID-19.

\section{Referências}

Brasil. Ministério da Saude, B. (2019). Vigitel Brasil 2018: Vigilância de fatores de risco e proteção para doenças crônic as por inquerito telefônico. In G. Estatística e Informação em Saúde.

Burden, S. J., Rademaker, J., Weedon, B. D., Whaymand, L., Dawes, H., \& Jones, A. (2020). Associations of Global Country Profiles and Modifiable Risk Factors with COVID-19 Cases and Deaths. SSRN Electronic Journal. https://doi.org/10.2139/ssrn.3627258

Caci, G., Albini, A., Malerba, M., Noonan, D. M., Pochetti, P., \& Polosa, R. (2020). COVID-19 and Obesity: Dangerous Liaisons. Journal of Clinical Medicine, 9(8), 2511. https://doi.org/10.3390/jcm9082511

Castilho, M. M., Westphal, G., Pereira, I. A. S., Martins, F. M., Bim, R. H., Thon, R. A., Moreira, V. F. R., Bevilaqua, C. A., Meireles, K. B., Okawa, R. T. P., \& Júnior, N. N. (2021). Teste de caminhada de 6 minutos (tc6m) na obesidade severa: considerações. Revista Valore, 5(e-555).

Castilho, M. M., Westphal, G., Pereira, I. A. S., Martins, F. M., Meireles, K. B., \& Nardo Junior, N. (2021). Efeitos de um programa multiprofissional de tratamento da obesidade no ambiente aquático em adolescentes acompanhados ou não de suas mães. Research, Society and Development, 10(1), e34010111929. https://doi.org/10.33448/rsd-v10i1.11929

Castilho, M. M., Westphal, G., Thon, R. A., Pereira, I. A. S., Martins, F. M., Amaral, M. F. do, Okawa, R. T. P., \& Nardo Junior, N. (2021). Efeitos de um programa multiprofissional de tratamento da obesidade no ambiente aquático em adultos com obesidade severa. Research, Society and Development, 10(1), e12910111636. https://doi.org/10.33448/rsd-v10i1.11636

Chen, P., Mao, L., Nassis, G. P., Harmer, P., Ainsworth, B. E., \& Li, F. (2020). Coronavirus disease (COVID-19): The need to maintain regular physical activity while taking precautions. Journal of Sport and Health Science, 9(2), 103-104. https://doi.org/10.1016/j.jshs.2020.02.001 
Christinelli, H. C. B., Westphal, G., Nardo Junior, N., Borim, M. L. C., Costa, M. A. R., \& Fernandes, C. A. M. (2021). Nutritional status and body composition in individuals with overweight or obesity using usual and unusual indicators. Research, Society and Development, 10(1), e4910111339. https://doi.org/10.33448/rsd-v10i1.11339

De Alves, T. A., Fernandes, J. A., Da Silva, C. S., Sousa, L. A., De Carvalho, A. B. L., Da Silva, I. Z. F., \& Pontes, J. A. F. (2016). Auto-percepção do papel do profissional de educação física no combate à obesidade: Um estudo piloto. Motricidade, 12, 30-41.

Dimer, N. A., Canto-Soares, N. do, Santos-Teixeira, L. dos, \& Goulart, B. N. G. de. (2020). Pandemia do COVID-19 e implementação de telefonoaudiologia para pacientes em domicílio: relato de experiência. CoDAS, 32(3), e20200144. https://doi.org/10.1590/2317-1782/20192020144

Direito, A., Carraça, E., Rawstorn, J., Whittaker, R., \& Maddison, R. (2017). mHealth Technologies to Influence Physical Activity and Sedentary Behaviors: Behavior Change Techniques, Systematic Review and Meta-Analysis of Randomized Controlled Trials. Annals of Behavioral Medicine, 51(2), 226-239. https://doi.org/10.1007/s12160-016-9846-0

Finer, N., Garnett, S. P., \& Bruun, J. M. (2020). COVID-19 and obesity. Clinical Obesity, 10(3). https://doi.org/10.1111/cob.12365

Flores-Mateo, G., Bamidis, P., Eugenio Santoro, E., \& DiFilippo, K. (2015). Mobile Phone Apps to Promote Weight Loss and Increase Physical Activity: A Systematic Review and Meta-Analysis. Journal of Medical Internet Research, 17(11).

Godino, J. G., Merchant, G., Norman, G. J., Donohue, M. C., Marshall, S. J., Fowler, J. H., Calfas, K. J., Huang, J. S., Rock, C. L., Griswold, W. G., Gupta, A., Raab, F., Fogg, B. J., Robinson, T. N., \& Patrick, K. (2016). Using social and mobile tools for weight loss in overweight and obese young adults (Project SMART): a 2 year, parallel-group, randomised, controlled trial. The Lancet Diabetes \& Endocrinology, 4(9), 747-755. https://doi.org/10.1016/S22138587(16)30105-X

Hartmann, C., \& Lopes, G. (2020). Reconhecimento Do Profissional De Educação Física Pelo Conselho Nacional De Saúde: Intervenção Recomendações Sobre Condutas E Procedimentos Na Atenção Básica À Saúde. In Revista Científica Cognitionis. https://doi.org/10.38087/2595.8801.12

Harzheim, E., Martins, C., Wollmann, L., Pedebos, L. A., Faller, L. de A., Marques, M. D. C., Minei, T. S. S., da Cunha, C. R. H., Telles, L. F., de Moura, L. J. N., Leal, M. H., Rodrigues, A. S., Rech, M. R. A., \& D'avila, O. P. (2020). Federal actions to support and strengthen local efforts to combat COVID-19: Primary health care (PHC) in the driver's seat. Ciencia e Saude Coletiva, 25, 2493-2497. https://doi.org/10.1590/1413-81232020256.1.11492020

Haufe, S., Kerling, A., Protte, G., Bayerle, P., Stenner, H. T., Rolff, S., Sundermeier, T., Kück, M., Ensslen, R., Nachbar, L., Lauenstein, D., Böthig, D., Bara, C., Hanke, A. A., Terkamp, C., Stiesch, M., Hilfiker-Kleiner, D., Haverich, A., \& Tegtbur, U. (2019). Telemonitoring-supported exercise training, metabolic syndrome severity, and work ability in company employees: a randomised controlled trial. The Lancet Public Health, 4(7), e343-e352. https://doi.org/10.1016/S2468-2667(19)30075-1

IBGE. (2018). PNAD Contínua TIC 2017: Internet chega a três em cada quatro domicílios do país. Agência IBGE notícias. https://agenciadenoticias.ibge.gov.br/agencia-sala-de-imprensa/2013-agencia-de-noticias/releases/23445-pnad-continua-tic-2017-internet-chega-a-tres-emcada-quatro-domicilios-do-pais

Kim, H. N., \& Seo, K. (2020). Smartphone-based health program for improving physical activity and tackling obesity for young adults: A systematic review and meta-analysis. International Journal of Environmental Research and Public Health, 17(1). https://doi.org/10.3390/ijerph17010015

Malta, D. C., Szwarcwald, C. L., Barros, M. B. de A., Gomes, C. S., Machado, Í. E., Souza Júnior, P. R. B. de, Romero, D. E., Lima, M. G., Damacena, G. N., Pina, M. de F., Freitas, M. I. de F., Werneck, A. O., Silva, D. R. P. da, Azevedo, L. O., \& Gracie, R. (2020). The COVID-19 Pandemic and changes in adult Brazilian lifestyles: a cross-sectional study, 2020. Epidemiologia e servicos de saude : revista do Sistema Unico de Saude do Brasil, $29(4)$, e2020407. https://doi.org/10.1590/S1679-49742020000400026

Mera, A., Tabares, E., Montoya, S., Muñoz, D., \& Monsalve, F. (2020). Recomendaciones prácticas para evitar el desacondicionamiento físico durante el confinamiento por pandemia asociada a COVID-19 Practical recommendations to avoid physical deconditioning during confinement due to pandemic associated with COVID-19. Universidad y Salud, 22(2), 166-177.

Monteiro, C. A. (2018). A Epidemiologia da Obesidade. 15-30.

Wolrd Health Organization (2018). The top 10 causes of death. https://www.who.int/en/news-room/fact-sheets/detail/the-top-10-causes-of-death

Pereira, I. A. S., Martins, F. M., Westphal, G., Castilho, M. M., \& Nardo Junior, N. (2021). Programas multiprofissionais de tratamento da obesidade com metas de perda de peso definidas: uma revisão sistemática da literatura. Research, Society and Development, 10(1), e24710111779. https://doi.org/10.33448/rsd-v10i1.11779

Petrilli, C., Jones, S., Yang, J., Rajagopalan, H., O’Donnell, L., Chernyak, Y., Tobin, K., Cerfolio, R., Francois, F., \& Horwitz, L. (2020). Factors associated with hospitalization and critical illness among 4,103 patients with COVID-19 disease in New York City. Bmj. https://doi.org/10.1101/2020.04.08.20057794

Romeo, A., Edney, S., Plotnikoff, R., Curtis, R., Ryan, J., Sanders, I., Crozier, A., \& Maher, C. (2019). Can smartphone apps increase physical activity? systematic review and meta-analysis. Journal of Medical Internet Research, 21(3). https://doi.org/10.2196/12053

Schoeppe, S., Alley, S., Van Lippevelde, W., Bray, N. A., Williams, S. L., Duncan, M. J., \& Vandelanotte, C. (2016). Efficacy of interventions that use apps to improve diet, physical activity and sedentary behaviour: A systematic review. International Journal of Behavioral Nutrition and Physical Activity, 13(1). https://doi.org/10.1186/s12966-016-0454-y

Soares, C. B., Hoga, L. A., Peduzzi, M., Sangaleti, C., Yonekura, T., Silva, D. R. A. D., Dutra, V. F. D., Oliveira, R. M. P., Zoltowski, A. P. C., Costa, A. B., Teixeira, M. A. P., Koller, S. H. S. H., Grau, D. E. F. E., Evidência, D. E. R. D. E., Souza, M. T. De, Dias, M., Carvalho, R. De, Ercole, F. F., Melo, L. S. de, ... Trevizan, M. A. (2010). Revisão Integrativa versus Revisão Sistemática. Reme: Revista Mineira de Enfermagem, 8(1), 102-106. https://doi.org/10.1590/S1679-45082010RW1134

Tong, H. L., Coiera, E., Tong, W., Wang, Y., Quiroz, J. C., Martin, P., \& Laranjo, L. (2019). Efficacy of a mobile social networking intervention in promoting 
Research, Society and Development, v. 10, n. 1, e49910112222, 2021 (CC BY 4.0) | ISSN 2525-3409 | DOI: http://dx.doi.org/10.33448/rsd-v10i1.12222

physical activity: Quasi-experimental study. JMIR mHealth and uHealth, 7(3). https://doi.org/10.2196/12181

Wang, Y., Xue, H., Huang, Y., Huang, L., \& Zhang, D. (2017). A Systematic Review of Application and Effectiveness of mHealth Interventions for Obesity. Advances in Nutrition, 8(3), 449-462.

Westphal, G., Baruki, S. B. S., Mori, T. A. de, Montebello, M. I. de L., \& Pazzianotto-Forti, E. M. (2020). Effects of Individualized Functional Training on the Physical Fitness of Women with Obesity. Lecturas: Educación Física y Deportes, 25(268), 61-75. https://doi.org/10.46642/efd.v25i268.2084

Westphal, G., Soares, G. S., Vespasiano, B. de S., Christinelli, H. C. B., Pereira, I. A. S., Castilho, M. M., Martins, F. M., \& Nardo Junior, N. (2021). Taxa metabólica basal de adolescentes com sobrepeso ou obesidade. Research, Society and Development, 10(1), e35010111964. https://doi.org/10.33448/rsdv10i1.11964

World Health Organization. (2015). Controlling the global obesity epidemic. World Health Organization, 7(1), 1-2. http://www.who.int/nutrition/topics/obesity/en/

World Health Organization (WHO). (2020). IHR Procedures concerning public health emergencies of international concern (PHEIC). https://www.who.int/ihr/procedures/pheic/en/ 